\title{
The Power-law Formalism as a Tool for Modeling Hormonal Systems
}

\author{
ALBERT SORRIBAS ${ }^{\mathrm{a}, *}$ and ANGEL GONZÁLEZ ${ }^{\mathrm{b},+}$ \\ ${ }^{a}$ Departament de Ciències Mèdiques Bàsiques, Universitat de Lleida, Av.Rovira Roure, 44, 25198-Lleida, Espanya; ${ }^{b}$ Departament de \\ Ciències Fisiològiques II. Universitat de Barcelona, Feixa Llarga s/n. 08907-L'Hospitalet de Llobregat, Barcelona, Espanya
}

(Received 6 July 1998; In final form 28 January 1999)

\begin{abstract}
Modeling a hormone system requires a number of simplifying assumptions. Often, the final conceptual model incorporates a number of aggregated processes that have no correspondence with a single enzyme-catalyzed reaction. In such cases, it is discussible using models based on classical biochemical kinetics rate laws that are valid only under specific conditions. The power-law formalism provides an alternative framework for building up a mathematical model in such cases. The resulting model is a set of ordinary differential equations with a special structure that allows efficient symbolic and numerical analysis of the system's properties. In these equations, the underlying rate-laws of each of the component processes are represented by a power-law that is an exact representation of the actual rate-law at the operating point. The particular form of these equations allows representation of a wide range of kinetic features without changing the basic power-law form. Moreover, its parameters have an immediate interpretation as apparent kineticorders and rate-constants. This is especially helpful for incorporating both quantitative and qualitative information in the process of model definition. This is particularly useful when detailed kinetic information concerning system's components is not available. In this paper we show the utility of the power-law approach in this context by deriving an illustrative model of a complex physiological system: the hypothalamus-anterior pituitarythyroid network. First, we derive a conceptual model that incorporates the key features of this system. Then, we derive an S-system model, one of the preferred variants within the power-law formalism, and we show its utility in exploring the system properties. The model qualitatively reproduces the response of normal, hyperthyroid, and hypothyroid patients to a clinical test involving a thyrotropin releasing hormone injection. Finally, we illustrate the utility of this modeling strategy for studying the system's response to different dynamic patterns of regulatory signals, and for exploring how altered dynamic patterns of stimulatory signals can cause pathological states.
\end{abstract}

${ }^{*}$ E-mail: Albert.Sorribas@cmb.udl.es

†E-mail: Angel@medicina.ub.es 


\section{INTRODUCTION}

The lack of a detailed experimental characterization of the system's components is an important limitation for building a mathematical model. The difficulty of collecting reliable experimental data increases when the target system is a conceptual model of a complex situation. In that case, many of the included reactions correspond to aggregated processes and the use of a traditional modeling approach based on classical biochemical kinetics has serious limitations. Even in the case that detailed in vitro kinetic data on isolated reactions were available, their extrapolation to the intact system conditions is arguable (Savageau, 1992, 1995a,b). Compartmental analysis offers, in principle, a way of addressing this kind of problems and has widely used for modeling physiological systems. However, when the involved processes include non-linear processes, such as saturable kinetics, and incorporate a number of regulatory signals, the usual tools become difficult to apply. In such cases, a modeling strategy based on first principles is of limited applicability and a more systemic-oriented approach is needed. This strategy should provide a tool for exploring the system properties so that several hypotheses on the system structure and parameters could be tested.

The power-law formalism provides a modeling tool that meets this goal. In this approach, a Taylor-series approximation in logarithmic space leads to a power-law representation that models the kinetic properties of the system's components. The systemic description results from the usual node equations in which the different rates are represented by power-laws. The special properties of the power-law approach allow representation of a wide range of kinetic processes by using the same mathematical function (Curto et al, 1995, 1997, 1998; Shiraishi and Savageau 1992a,b,c,d; Ni and Savageau, 1996a,b). Furthermore, complex non-linear kinetics can be incorporated using two strategies. First, they can be approximated by the appropriate power-law at the operating point. Second, recasting the original function into a power-law set of differential equations we obtain an exact representation of the original function (Voit 1993). Power-law models offer several advantages: (i) They provide a systematic mathematical representation for non-linearities, including the possibility of incorporating complex dynamic patterns; (ii) A reliable quantitative model can incorporate both quantitative and qualitative information; and (iii) The system's properties can be analyzed both numerically and algebraically (see Voit, 1991 and references therein for a complete account of this technique).

Power-law based models, either as S-system or GMA (Generalized Mass Action) models, have been successfully used for modeling complex metabolic systems (Voit, 1991; Shiraishi and Savageau, 1992a,b,c,d; Savageau, 1992, 1995; Cascante et al., 1995; Sorribas et al., 1995; $\mathrm{Ni}$ and Savageau, 1996a,b; Curto et al., 1995, 1997). The power-law formalism provides also a theoretical framework for discussing the design principles of a metabolic system through the method known as Controlled Mathematical Comparisons. This method has been successfully applied to investigate the design principles of gene circuits and immune networks (Hlavaceck and Savageau, 1996, and references therein). As any modeling procedure, the power-law formalism incorporates the possibility of studying the system's dynamic behavior. Moreover, complex dynamic patterns can be incorporated as forcing functions into power-law models using the recasting technique (Voit, 1993). This produces a new model that retains the power-law structure. The ability of maintaining the same mathematical structure is a fundamental property of this class of models.

In this paper, we show the utility of the powerlaw formalism in modeling a metabolic pathway from which a poor characterization is available. As an example, we derive an illustrative model of the hypothalamus-anterior pituitary-thyroid network. In this system, the pattern of temporal variation in hormone levels defines the healthy state, and pathological situations are associated to changes in these patterns. Therefore, understanding hormonal regulation and its pathological manifestations requires relating dynamic behavior and regulatory influences. 
However, a detailed description of all the underlying processes is often difficult, and the modeling process requires some simplifications that result in aggregation of processes. This makes it difficult to kinetically characterize the system and requires using an appropriate modeling strategy.

The hypothalamus-anterior pituitary-thyroid network includes many regulatory signals between the different glands and related hormones. The function of the thyroid gland is mainly regulated by the anterior pituitary hormone thyrotropin (TSH) (Magner, 1990; Brabant et al., 1989). TSH is primarily regulated by the hypothalamus through release of thyrotropin releasing hormone (TRH). Thyroid hormone levels also help control TSH by means of a negative feedback mechanism (Jackson, 1982; Morley, 1981). Besides this central regulation, periferic regulation, finer and specific for each tissue, help tuning thyroid activity according to the needs of the different organs and systems in front of changes in the internal or external medium (Oppenheimer et al., 1987).

Dysfunction of the thyroid gland leads to different pathological situations. The hyperfunction and its clinical manifestations are the result of the action upon the tissues of supernormal quantities of $T_{3}$ and $T_{4}$. In the hypofunction of the thyroid, the syndromes result from the absence of normal levels of thyroid hormones. The hormonal deficit affects all the tissues, leading to a wide range of clinical repercussions. Although the clinical symptoms have been identified, the ultimate cause of specific pathological states is under discussion.

A detailed mathematical model incorporating all the relevant processes and regulatory signals would be helpful for discussing the origin of these pathological situations. However, such a model is difficult to define provided the complexity of the different processes involved and the lack of specific information on their kinetic properties. Previous models of thyroid metabolism have focused on the distribution of hormones in different body compartments through the analysis of injected radioactive tracers (see for instance Hays et al., 1988; Jacobs et al., 1997). Hatakeyama and Yagi (1985) proposed a model based on linear kinetics. This model was able of reproducing key features of the system, including observations related to Graves' disease.

Definition of more complete models is difficult for many reasons. Mainly, it is difficult to build up a reliable kinetic description of the involved processes. Although first order kinetics may be a good election in some cases, when regulatory signals are present one must consider alternative kinetic representations. The power-law formalism offers an alternative in such cases. In the following, we will use this approach for building an illustrative model of thyroid metabolism, including periodic and chaotic variations in the external signals. Details on the hypothalamus-anterior pituitary-thyroid network and the rationale for the conceptual model in Figure 1 are discussed in Appendix 1. In Section 2, an Ssystem model is derived for studying this pathway. Using this model, we discuss the steady-state response of the system to external variables, and the way in which dynamic patterns of these variables can be used for testing the system behavior. In Appendix 2, we discuss the parameter values used for testing this response. In Section 3, we show that our model reproduces clinical observations both in normal as simple pathological situations. The model response to a chaotic stimulus is used to explore a potential source of hyperthyroidism. Our results instruct on the possibilities of power-law modeling and provide a starting point for considering more realistic models of this system.

\section{POWER-LAW MODELING}

\subsection{Modeling Strategy: The S-System}

A conceptual model for the hypothalamus-anterior pituitary-thyroid network is shown in Figure la (see Appendix 1 for details). A first step in defining a mathematical model for this scheme is to translate the included metabolites and reactions into an appropriate numbering scheme. The scheme in Figure $1 \mathrm{~b}$ shows how this is done in our case. For an easy reference, in Table I we list the correspondences between metabolites and variables. Once this 


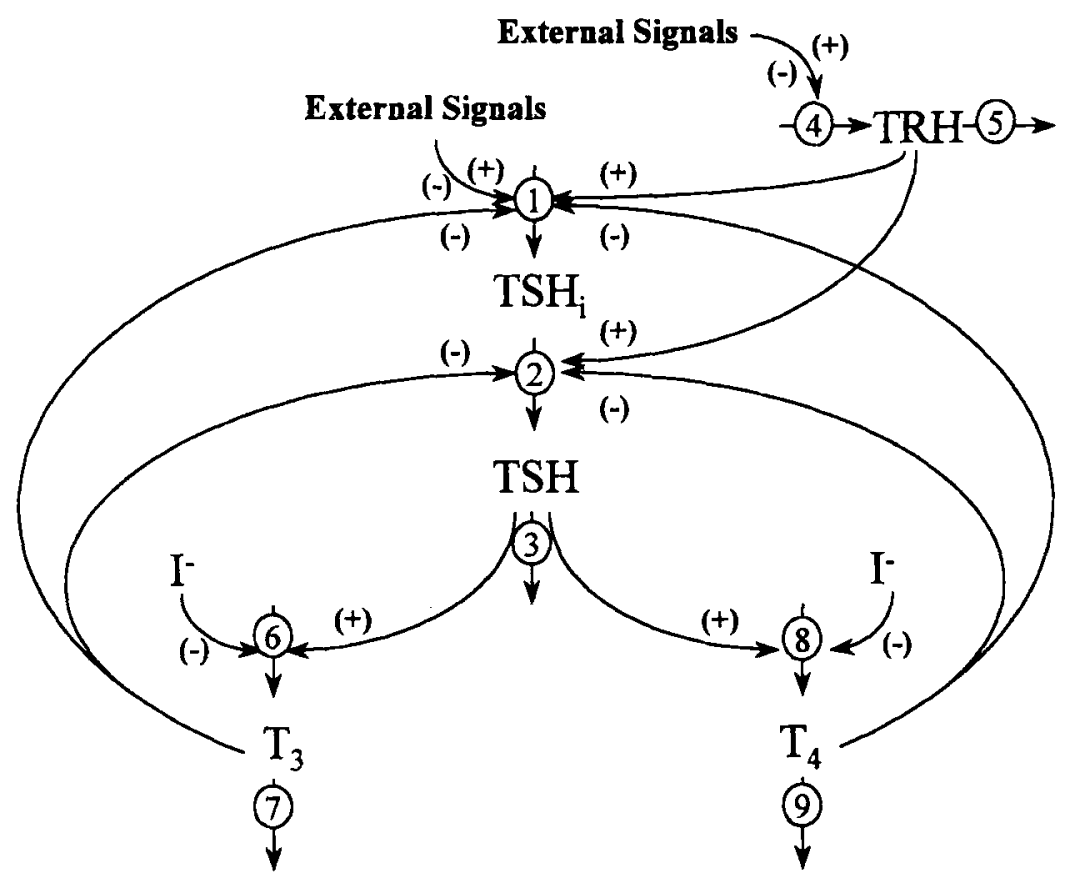

(a)

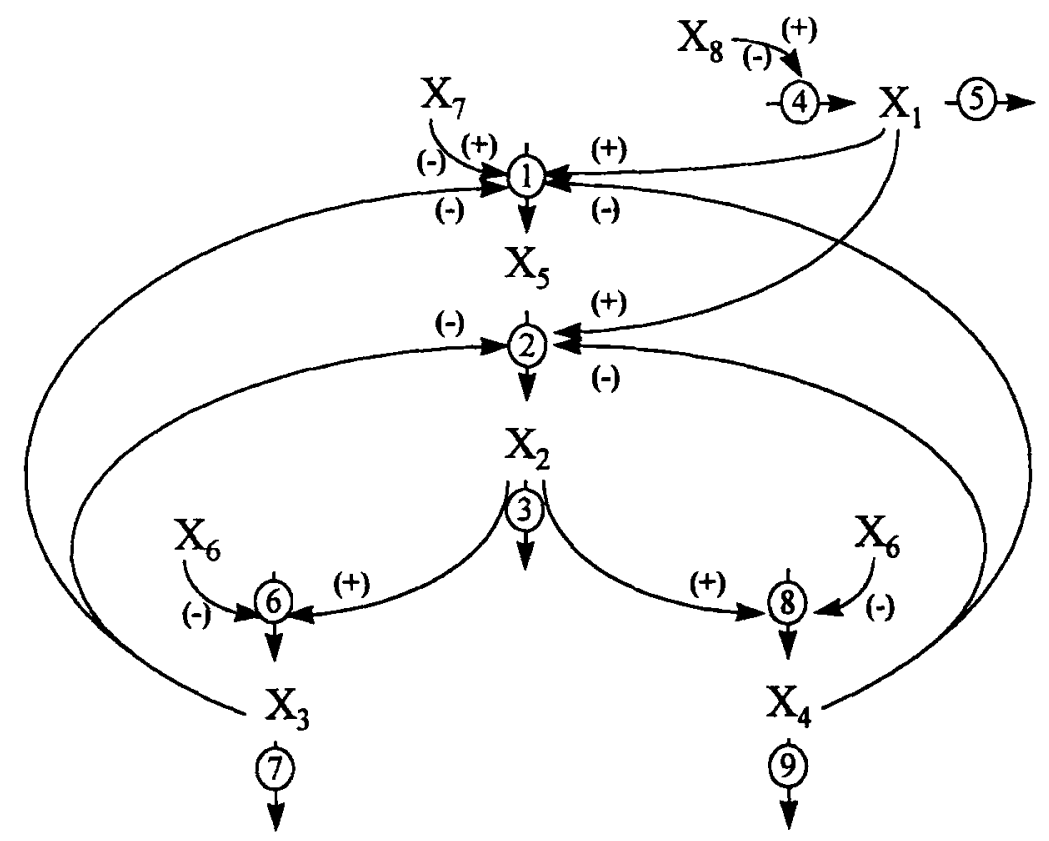

(b)

FIGURE 1 Model of thyroid hormone metabolism in man. (a) Conceptual model; (b) Numbering scheme. For details see Appendix 1. 
TABLE I Correspondence between metabolites and variable numbers in the model of the hypothalamus-anterior pituitarythyroid network (Figure 1). See Appendix 1 for details

\begin{tabular}{lc}
\hline Metabolite & Variable \\
\hline TRH & $X_{1}$ \\
TSH & $X_{2}$ \\
$T_{3}$ & $X_{3}$ \\
$T_{4}$ & $X_{4}$ \\
TSH $_{\mathrm{i}}$ & $X_{5}$ \\
Iodine & $X_{6}$ \\
Stimulatory or inhibitory signal acting & $X_{7}$ \\
$\quad$ on the anterior pituitary & $X_{8}$ \\
Stimulatory or inhibitory signal acting & \\
on the hypothalamus & \\
\hline
\end{tabular}

numbering scheme has been established, a mathematical model can be written as a set of ordinary differential equations expressing the balance of the different processes in the time change of the involved hormones and metabolites. This produces:

$$
\begin{aligned}
& \dot{X}_{1}=v_{4}-v_{5}=V_{1}^{+}-V_{1}^{-} \\
& \dot{X}_{2}=v_{2}-v_{3}=V_{2}^{+}-V_{2}^{-} \\
& \dot{X}_{3}=v_{6}-v_{7}=V_{3}^{+}-V_{3}^{-} \\
& \dot{X}_{4}=v_{8}-v_{9}=V_{4}^{+}-V_{4}^{-} \\
& \dot{X}_{5}=v_{1}-v_{2}=V_{5}^{+}-V_{5}^{-}
\end{aligned}
$$

In this equation, $V_{i}^{( \pm)}$represents the synthesis or degradation of $X_{i}$, and $\dot{X}_{i}$ indicates a time derivative. The corresponding rate expressions for these processes are functions of several variables with the general form:

$$
V_{i}^{( \pm)}=f_{i}^{( \pm)}\left(X_{1}, \ldots, X_{8}\right) \quad i=1, \ldots, 5
$$

According to the discussion above, most of the processes represented in this model do not correspond to single enzymatic reactions. In this situation, the actual expression for $f_{i}^{( \pm)}\left(X_{1}, \ldots, X_{8}\right)$ is unknown, and the use of kinetic rate-laws, for example Michaelis-Menten-like equations, is arguable (Savageau 1992, 1995a). For instance, in our scheme the release of TSH is $V_{2}^{+}$, which corresponds to $\nu_{2}$. This process depends on $\mathrm{TSH}_{i}$, and is inhibited by the levels of $T_{3}$ and $T_{4}$ and activated by TRH.
Then, the corresponding rate-law for representing this process would be:

$$
V_{2}^{+}=f\left(X_{1}, X_{3}, X_{4}, X_{5}\right)
$$

Which would be an appropriate choice for representing the rate-law $f\left(X_{1}, X_{3}, X_{4}, X_{5}\right)$ ? It is clear that being an aggregated process, the choice in not easy. Should we use a Michaelis-Menten equation when the represented process is not a single enzymatic process? Previous experience with the analysis of complex systems suggest that the power-law formalism is adequate for representing aggregated rate-laws (a complete discussion of this idea can be found in: Sorribas and Savageau, 1989; Shiraishi and Savageau, 1992a,b,c,d; Ni and Savageau, 1996a,b; Curto et al, 1997). Following this methodology, each process is represented by a power-law function that includes a rate constant and a product of power functions, one for each metabolite or effector that affects the rate of the target process. In each case, the exponent is the apparent kinetic-order for the metabolite in the considered process. Then, using the numbering scheme in Figure 1, the power-law representing $V_{2}^{+}$is:

$$
V_{2}^{+}=\alpha_{2} X_{1}^{g 21} X_{3}^{g_{22}} X_{4}^{g_{24}} X_{5}^{g 25}
$$

Parameters $g_{i j}$ are called kinetic-orders, and correspond to the local sensitivity of the synthesis of the $i$ th metabolite with respect the $j$ th variable at a given operating point indicated by the subscript 0 :

$$
g_{i j}=\left(\frac{\partial V_{i}^{+}}{\partial X_{j}}\right)_{0} \frac{X_{j 0}}{V_{i 0}^{+}}
$$

Then, a kinetic-order quantifies the effect of a small change on a variable on the rate of a given process. If an increase in $X_{j}$ produces an increase in the rate of the process, then at a given point the ratelaw will have a positive slope yielding a positive kinetic-order. On the contrary, if an increase in $X_{j}$ produces a decrease in the rate of the considered process, i.e. $X_{j}$ is an inhibitor, then a negative slope will produce a negative kinetic-order. According to this definition, if we recall that $T_{3}$ and $T_{4}$ (i.e. $X_{3}$ and $X_{4}$ ) are inhibitors of the TSH release, we include 
power-law terms for $X_{3}$ and $X_{4}$ with negative kinetic orders $g_{23}$ and $g_{24}$. These kinetic orders have a negative value since they quantify an inhibitory effect on the considered process. Following the same rationale, we include an $X_{5}$ power term with a positive kinetic order $g_{25}$. This parameter is positive because $X_{5}$ is the substrate of the reaction. Finally, we include an $X_{1}$ power term with its corresponding kinetic order $g_{21}$. This parameter is positive because TRH acts as an activator of the release of TSH. Parameter $\alpha_{2}$ is called rate constant, and its meaning is immediate from Equation 4.

The kinetic-orders of the power-law formalism can be interpreted in useful ways if we consider its meaning. For example, consider the expression $V_{2}^{+}=\alpha_{2} X_{1}^{g_{21}} X_{3}^{g_{23}} X_{4}^{g_{24}} X_{5}^{g_{25}}$. If at a given concentration, $X_{3}$ inhibits a $50 \%$ of the synthesis of $X_{2}$, then we can consider $X_{3}^{g_{23}}=1-0.5 \rightarrow g_{23}=$ $0.5 / \log \left(X_{3}\right)$ (see Curto et al. (1998) for details). This is very useful for tentatively estimating a kinetic order in complex systems. Furthermore, if we recall the definition of a kinetic-order, then a process close to saturation by $X_{j}$ should have a kineticorder close to zero, while it is possible to show that kinetic-orders greater than zero are only possible for cooperative processes. A process with a MichaelisMenten rate law has a kinetic-order between zero, if it is saturated, and one, when the substrate is well below the $K_{m}$. If the substrate is equal to the $K_{m}$, then the kinetic-order is 0.5 . As a particular case, the power-law formalism includes linear kinetics. This corresponds to set the kinetic-order equal to one (see Appendix 2).

The theory behind the power-law representation, which is based on a Taylor series approximation in logarithmic space (Savageau, 1972; Voit and Savageau, 1987; Sorribas and Savageau, 1989), assures that Equation 4 is a good approximation to the actual unknown function. Convergence is guaranteed in the vicinity of an operating point. More important, when the whole system is represented using this strategy, the predictions on the systemic behavior are accurate over a wide range, provided the model incorporates all the relevant processes (Voit and Savageau, 1987; Sorribas and Savageau, 1989). This is an interesting property that makes this kind of modeling strategy useful for studying metabolic pathways.

Applying this strategy, the resulting model for the thyroid metabolism scheme in Figure 1b is:

$$
\begin{aligned}
& \dot{X}_{1}=\alpha_{1} X_{8}^{g_{18}}-\beta_{1} X_{1}^{h_{11}} \\
& \dot{X}_{2}=\alpha_{2} X_{1}^{g_{21}} X_{3}^{g_{23}} X_{4}^{g_{24}} X_{5}^{g_{25}}-\beta_{2} X_{2}^{h_{22}} \\
& \dot{X}_{3}=\alpha_{3} X_{2}^{g_{32}} X_{6}^{g_{36}}-\beta_{3} X_{3}^{h_{22}} \\
& \dot{X}_{4}=\alpha_{4} X_{2}^{g_{42}} X_{6}^{g_{46}}-\beta_{4} X_{4}^{h_{44}} \\
& \dot{X}_{5}=\alpha_{5} X_{1}^{g_{51}} X_{3}^{g_{53}} X_{4}^{g_{54}} X_{7}^{g_{57}}-\alpha_{2} X_{1}^{g_{21}} X_{3}^{g_{23}} X_{4}^{g_{24}} X_{5}^{g_{25}}
\end{aligned}
$$

This equation has an S-system structure. Each dependent variable has a single term for synthesis, and a single term for degradation. A GMA model would result if more than one term were present either for synthesis or degradation. Following the usual nomenclature in S-system models, the kinetic orders that refer to a degradation term are expressed as $h_{i j}$, and the corresponding rate constant as $\beta_{i}$. In this model, the synthesis of $X_{2}(\mathrm{TSH})$ and the release of $X_{5}\left(\mathrm{TSH}_{i}\right)$ are the same process. Then in Equation 6 we use the same power-law for both processes.

\subsection{Dimensional Analysis}

The model in Equation 6 can be reformulated to reduce the number of parameters (Lin and Segel, 1974). In the case of power-law models a useful strategy is to define $F_{i}=V_{i 0} / X_{i 0}$ and $u_{i}=X_{i} / X_{i 0}$. With this change, the model can be expressed in terms of the relative concentration with respect to the reference steady-state (Hlavaceck and Savageau, 1996):

$$
\begin{aligned}
& \dot{u}_{1}=F_{1}\left(u_{8}^{g_{14}}-u_{1}^{h_{11}}\right) \\
& \dot{u}_{2}=F_{2}\left(u_{1}^{g_{21}} u_{3}^{g_{23}} u_{4}^{g_{24}} u_{5}^{g_{25}}-u_{2}^{h_{22}}\right) \\
& \dot{u}_{3}=F_{3}\left(u_{2}^{g_{32}} u_{6}^{g_{36}}-u_{3}^{h_{33}}\right) \\
& \dot{u}_{4}=F_{4}\left(u_{2}^{g_{42}} u_{6}^{g_{46}}-u_{4}^{h_{44}}\right) \\
& \dot{u}_{5}=F_{5}\left(u_{1}^{g 51} u_{3}^{g 53} u_{4}^{g_{54}} u_{7}^{g_{57}}-u_{1}^{g_{21}} u_{3}^{g_{23}} u_{4}^{g_{24}} u_{5}^{g_{25}}\right)
\end{aligned}
$$

In this representation, the $F_{i}$ terms denote the turnover of $X_{i}$. It is convenient to consider a relative 
turnover, instead of absolute values. This can be achieved by replacing time by $\tau=t . F_{2}$. We obtain:

$$
\begin{aligned}
& u_{1}^{\prime}=F_{1} / F_{2}\left(u_{8}^{g_{18}}-u_{1}^{h_{11}}\right) \\
& u_{2}^{\prime}=u_{1}^{g_{21}} u_{3}^{g_{23}} u_{4}^{g_{24}} u_{5}^{g_{25}}-u_{2}^{h_{22}} \\
& u_{3}^{\prime}=F_{3} / F_{2}\left(u_{2}^{g_{32}} u_{6}^{g_{36}}-u_{3}^{h_{33}}\right) \\
& u_{4}^{\prime}=F_{4} / F_{2}\left(u_{2}^{g_{42}} u_{6}^{g_{46}}-u_{4}^{h_{44}}\right) \\
& u_{5}^{\prime}=F_{5} / F_{2}\left(u_{1}^{g_{51}} u_{3}^{g_{53}} u_{4}^{g_{54}} u_{7}^{g_{55}}-u_{1}^{g_{21}} u_{3}^{g_{23}} u_{4}^{g_{24}} u_{5}^{g_{25}}\right)
\end{aligned}
$$

where $u_{i}^{\prime}=\left(\left(d u_{i}\right) /(d \tau)\right)$. In this equation, the turnover of TSH has been considered as a reference for all the remainder. The final version of our model, Equation 8, is more convenient for discussing the dynamic response to different perturbations on the external signals.

\subsection{Expected Dynamic Behavior}

By analyzing the corresponding logarithmic-gains, it can be shown that the model in Equation 6 (now redefined in Equation 8) qualitatively agrees with the expected steady-state behavior after a sustained increase in an external signal (see Appendix 3). The concept of logarithmic-gain, however, predicts the new steady-state values of a dependent variable in response to a sustained increase in an independent variable. Logarithmic gains do not provide a picture of the dynamic response between both steady states.

Transient responses play an important role in characterizing this system. The dynamic response of TSH levels after a TRH dose $(200-300 \mu \mathrm{g}$ in an intravenous bolus) is used for evaluating the functional status of an individual. This test (Brabant et al, 1990a) explores the functionality of the hypothalamic-hypophysis-thyroid system. TSH increases in normal conditions when the hypothalamus cannot segregate TRH, especially in absence of thyroid hormones which leads to an hyperactivity of the hypophysis. TSH does not change when hypophysis activity is low, especially due to an excess level of thyroid hormones.

In normal conditions, external signals act following complex dynamic patterns. Normal levels of thyroid hormones are a result of such patterns.
Pathological levels can be produced both by changes in the internal structure of the system and by changes in the pattern of the external signals. Changes in TSH, TRH and thyroid hormones patterns have been measured in relation with pathological states of different origins. Examples are changes associated to sleep depravation, major depression, and HIV infection (Rondanelli et al. 1997; Leproult et al. 1997; Sullivan et al; 1997; Campos-Barros et al., 1997). It would be interesting to investigate the effect of different stimulatory patterns on the hypothalamicpituitary-thyroid axis to discuss the possible relationship between these patterns and pathological patterns of thyroid hormones.

Conceptually, one can consider two extreme situations as models for representing the dynamics of external signals: regular cyclic variations (which could approximate circadian rhythms), and chaotic variations (which can mimic complex patterns in external signals that can model pathological conditions). Variation in external signals will be simulated using these two extreme possibilities. These patterns are incorporated into the model following the methodology defined by Voit (1993) after recasting the desired pattern into power-law equations (see below).

\subsection{Representation of Dynamic Patterns for External Signals}

\subsubsection{Sinusoidal pattern}

External stimulus in thyroid hormone metabolism comprises many different patterns (Brabant, 1990b; Custro and Scaglione 1980). A common feature of these patterns is a temporal variation that ranges from regular circadian rhythms to chaotic spike patterns. Mathematical representation of these patterns can be achieved by relatively simple non-linear equations or by systems of non-linear differential equations.

Power-law models can incorporate any kind of external perturbation represented by a non-linear function or by a set of ordinary differential equation. Although it is not strictly necessary, the best strategy 
is to recast the original functions into power-law equations (Voit, 1993). The advantage of this procedure is a final model in power-law form that can be efficiently simulated with special algorithms that takes advantage of the S-system or GMA structures. As a simple example, consider a sinusoidal pattern. A function representing such pattern could be:

$$
u(t)=\frac{\operatorname{Sin}\left[t^{a} / b\right]+1}{c}+1
$$

This function assures a variation between 1 , the basal value for $u(t)$, and $1+2 / c$. The parameters $a$ and $b$ change the frequency of the considered signal, and $c$ the amplitude (Figure 2). This function may be incorporated into our model in substitution of $u_{7}$

(a)

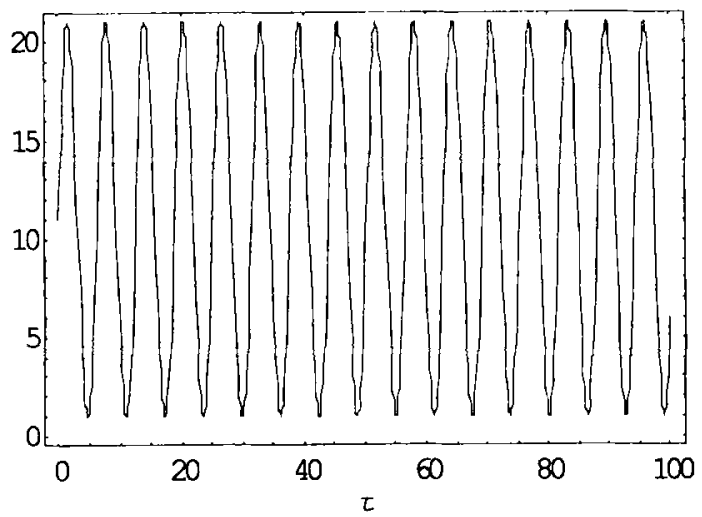

(c)

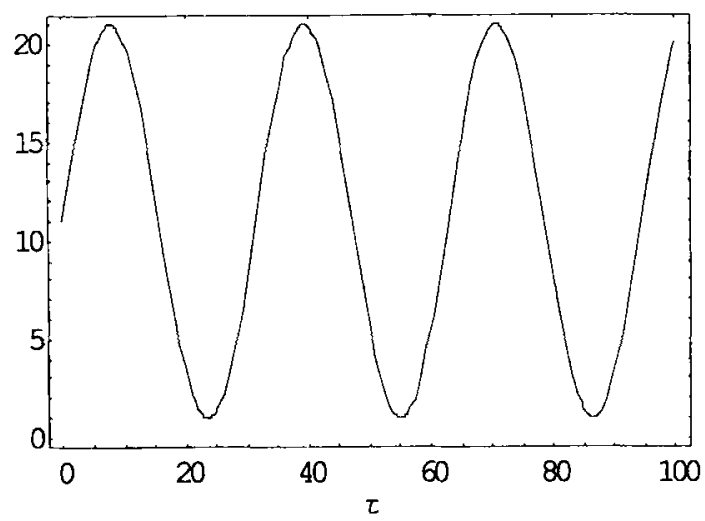

or $u_{8}$ providing the desired model for a regular circadian rhythm. As suggested by Voit (1993), it is convenient to recast Equation 9 as an S-system differential equation in order to incorporate this signal into the original model. This can be accomplished by the following procedure. First, we call $y_{1}$ the independent variable. Second we introduce a new variable $y_{2}=y_{1}^{a} / b$, and call $y_{3}$ the original function (Equation 9). Differentiation and introduction of a fourth variable $y_{4}$ leads to the following system of differential equations:

$$
\begin{array}{ll}
y_{1}(\tau)=\tau & \Rightarrow y_{1}^{\prime}=1 \\
y_{2}(\tau)=y_{1}^{a} / b & \Rightarrow y_{2}^{\prime}=\frac{a}{b} y_{1}^{a-1}
\end{array}
$$

(b)

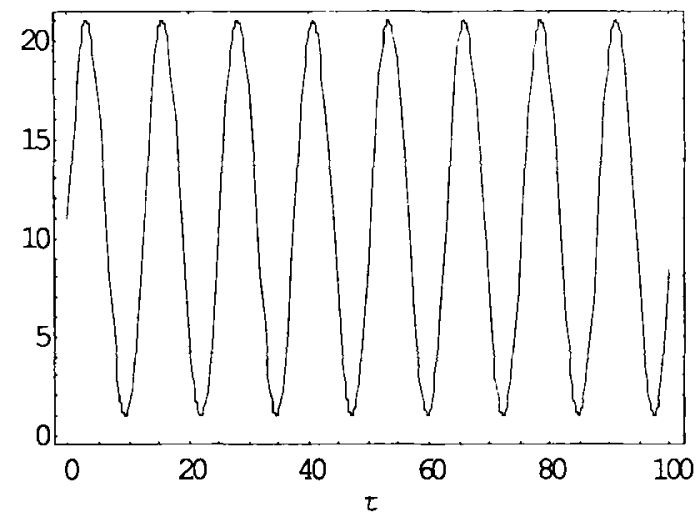

(d)

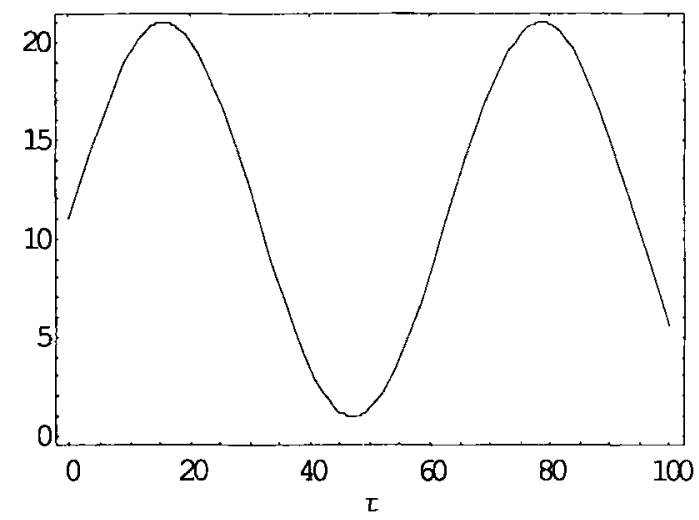

FIGURE 2 Sinusoidal patterns obtained with the recasted version (Equations $17-18$ ) of the sinusoid function $u(\tau)=\frac{\operatorname{Sin}\left[\tau^{a} / b\right]+1}{c}+1$. In all cases: $a=1, c=0.1$, and: (a) $b=1$; (b) $b=2$; (c) $b=5$; (d) $b=10$. 


$$
\begin{aligned}
& y_{3}(\tau)=\frac{\operatorname{Sin}\left(y_{2}\right)}{c}+\frac{1}{c}+1 \Rightarrow y_{3}^{\prime}=\frac{\operatorname{Cos}\left(y_{2}\right)}{c} \dot{y}_{2} \\
& y_{4}(\tau)=\frac{\operatorname{Cos}\left(y_{2}\right)}{c} \quad \Rightarrow y_{4}^{\prime}=-\frac{\operatorname{Sin}\left(y_{2}\right)}{c} \dot{y}_{2}
\end{aligned}
$$

where we take $\tau$ as time. Substitution produces the final equations:

$$
\begin{aligned}
y_{1}^{\prime} & =1 \\
y_{2}^{\prime} & =\frac{a}{b} y_{1}^{a-1} \\
y_{3}^{\prime} & =\frac{a}{b} y_{1}^{a-1} y_{4} \\
y_{4}^{\prime} & =\left(\frac{1}{c}+1\right) \frac{a}{b} y_{1}^{a-1}-\frac{a}{b} y_{1}^{a-1} y_{3}
\end{aligned}
$$

where $y_{3}$ is the original sinusoidal signal (Equation 9). The required initial conditions are:

$$
\begin{aligned}
& y_{1}\left(\tau_{0}\right)=\tau_{0} \\
& y_{2}\left(\tau_{0}\right)=\frac{\tau_{0}^{a}}{b} \\
& y_{3}\left(\tau_{0}\right)=\frac{\operatorname{Sin}\left(\tau_{0}^{a} / b\right)}{c}+\frac{1}{c}+1 \\
& y_{4}\left(\tau_{0}\right)=\frac{\operatorname{Cos}\left(\tau_{0}^{a} / b\right)}{c}
\end{aligned}
$$

The structure of Equation 11 corresponds to an S-system model, which allows introducing the variation of an independent variable within our thyroid hormone model maintaining the same mathematical structure. For instance, the set of equations that will allow investigating the system's response to a sinusoidal stimulus in $u_{8}$ are obtained by incorporating Equations (11-12) to Equation (8) and by changing $u_{8}$ by $y_{3}$ :

$$
\begin{aligned}
& u_{1}^{\prime}=F_{1} / F_{2}\left(y_{3}^{g_{18}}-u_{1}^{h_{11}}\right) \\
& u_{2}^{\prime}=u_{1}^{g_{21} 1} u_{3}^{g_{23} 3} u_{4}^{g_{24}} u_{5}^{g^{25}}-u_{2}^{h_{22}} \\
& u_{3}^{\prime}=F_{3} / F_{2}\left(u_{2}^{g_{32}} u_{6}^{g_{36}}-u_{3}^{h_{33}}\right) \\
& u_{4}^{\prime}=F_{4} / F_{2}\left(u_{2}^{g_{42}} u_{6}^{g_{46}}-u_{4}^{h_{44}}\right) \\
& u_{5}^{\prime}=F_{5} / F_{2}\left(u_{1}^{g 51} u_{3}^{g 53} u_{4}^{g 54} u_{7}^{g_{57}}-u_{1}^{g_{21}} u_{3}^{g_{23}} u_{4}^{g_{24}} u_{5}^{g_{25}}\right) \\
& y_{1}^{\prime}=1
\end{aligned}
$$

$$
\begin{aligned}
& y_{2}^{\prime}=\frac{a}{b} y_{1}^{a-1} \\
& y_{3}^{\prime}=\frac{a}{b} y_{1}^{a-1} y_{4} \\
& y_{4}^{\prime}=\left(\frac{1}{c}+1\right) \frac{a}{b} y_{1}^{a-1}-\frac{a}{b} y_{1}^{a-1} y_{3}
\end{aligned}
$$

\subsubsection{Chaotic pattern}

External signals acting as stimulus in thyroid hormone metabolism can follow periodic patterns of variation that are more closely related to chaotic than to regular behavior. Typically, stimulus raises over its basal values with a quick return to this basal state in cycles that are repeated with different amplitudes and with random firing times. It would be interesting to evaluate the performance of our model under such class of dynamic behavior. Voit (1993) suggested a way of incorporating chaotic dynamics for the external variables in a power-law model. We have adapted this method to obtain the patterns showed in Figure 3.

There are many different equations that could be used to generate a chaotic signal. We will use the modified screw-type Rössler-Band defined by Voit (see Voit, 1993, for details). The set of equations is:

$$
\begin{aligned}
y_{1}^{\prime}= & y_{2}-q-y_{3}+r \\
y_{2}^{\prime}= & -y_{1}+p-a\left(q-y_{2}\right) \\
y_{3}^{\prime}= & (p q-p b+c q)-(q-b) y_{1}+ \\
& y_{1} y_{3}-(c+p) y_{3}
\end{aligned}
$$

with parameters: $p=25, q=50, r=50, a=0.5$, $b=0.4$, and $c=4.5$. This equation is a GMA set of equations and could be recasted into an equivalent $S$-system if required. We will use the GMA equations for simplicity. Appropriate initial values are: $y_{1}(0)=25, y_{2}(0)=47, y_{3}(0)=1$. In order to obtain a variation with a minimum value of unity, we substitute the target signal as: $u_{k}=$ $\left(y_{2} / 47\right)^{n}$, if the desired behavior is a chaotic cycle (Figure 3a), or $u_{k}=\left(y_{3} / 50\right)^{n}$, if we want to model a spike-like behavior (Figure $3 b$ ). 


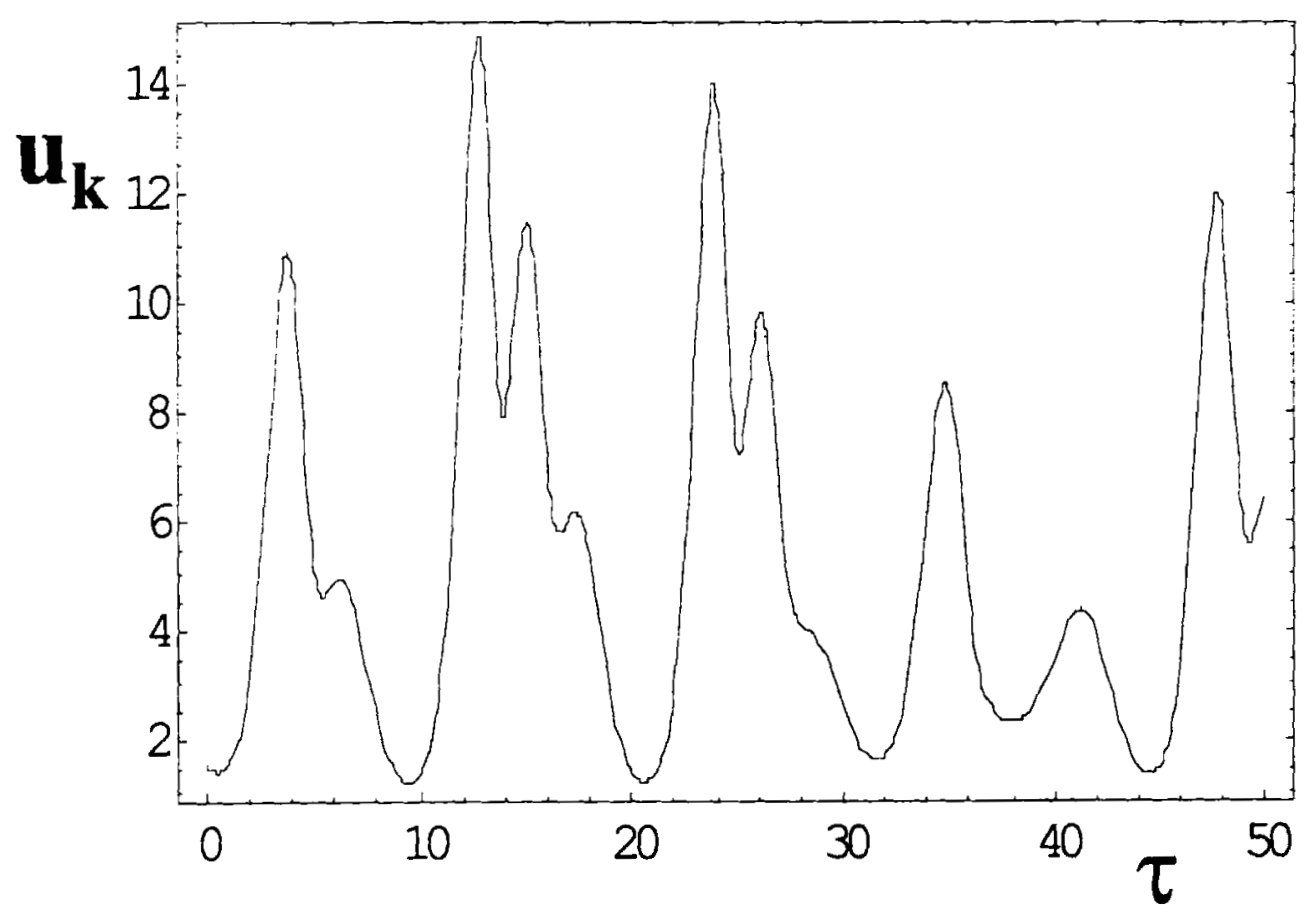

(a)

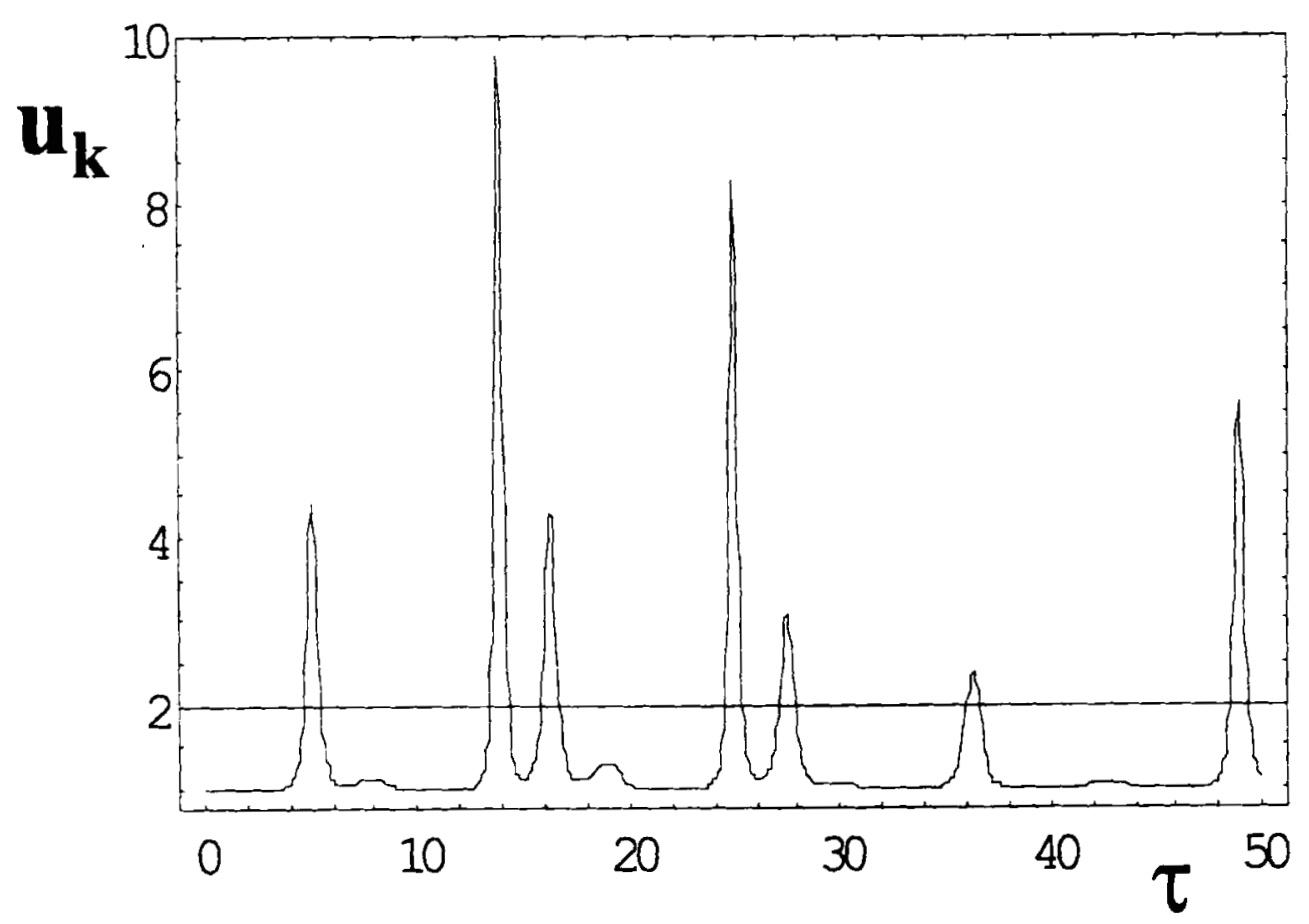

(b)

FIGURE 3 (a) Chaotic signals generated by the Equation (20) and the transformation $u_{k}=\left(y_{2} / 47\right)^{10}$. (b) Spike-like behavior generated by Equation (20) and the transformation $u_{k}=\left(y_{3} / 50\right)^{6}$. 


\section{MODEL COMPUTER SIMULATIONS}

\subsection{TRH Test in Normal Subjects}

The functionality of the hypothalamus-hypophysisthyroid gland system is tested using the TRH test (Brabant et al, 1990a). This consists in an intravenous injection of TRH $(200-300 \mu \mathrm{g})$, and measurement of TSH and thyroid hormones levels at different times. A typical response in normal subjects results in a transient raise in TSH half an hour after injection. Maximum values of 4-5-fold increase comparing to its basal level are observed. Normal values are recovered in 5-10 minutes. TSH raise is followed by an increase in thyroid hormones that can double their basal level. Basal levels are recovered 1-2 hours after TRH injection.

To simulate this situation we need to consider appropriate parameter values. However, an accurate estimation of the parameter set corresponding to Equation 8 is difficult. Previous models of the same system can provide a clue for some of the parameters (see Hatakeyama and Yagi, 1985), but in many cases we found no clear experimental evidence for some of the included processes. In Appendix 2, we present the rationale behind the parameter set we use in our simulations (Table II).

Although the parameter set has been obtained from qualitative considerations, results shown in Figure 4 qualitatively reproduce the typical outcome after a TRH test. In normal subjects (Figure 4a), the expected behavior is obtained after injection of a TRH dose $10^{7}$ higher than the basal levels. This value is justified if we recall that basal levels of TRH reduce to few molecules and that the injection dose is $200-300 \mu \mathrm{g}$.

\subsection{TRH Test in Hyperthyroid and Hypothyroid Patients}

Patients suffering from primary hyperthyroidism show a transient increase in TSH that doubles its basal level. This is due to the high levels of $T_{3}$ and $T_{4}$ (10-100 times higher than the normal basal values) and their inhibitory effect on TSH synthesis and
TABLE II Parameter values used for simulating the response of normal subjects (see Appendix 2 for details)

\begin{tabular}{|c|c|}
\hline \multicolumn{2}{|l|}{ Relative turnover numbers } \\
\hline $\begin{array}{l}F_{1} / F_{2} \\
F_{3} / F_{2} \\
F_{4} / F_{2} \\
F_{5} / F_{2}\end{array}$ & $\begin{array}{l}1.0 \\
0.05 \\
0.05 \\
0.1\end{array}$ \\
\hline \multicolumn{2}{|c|}{ Effect of TRH on TSH synthesis and release } \\
\hline $\begin{array}{l}g_{51} \\
g_{21}\end{array}$ & $\begin{array}{l}0.5 \\
0.1\end{array}$ \\
\hline \multicolumn{2}{|c|}{ Effect of TSH on $T_{3}$ and $T_{4}$ synthesis and release } \\
\hline $\begin{array}{l}g_{32} \\
g_{42}\end{array}$ & $\begin{array}{l}0.5 \\
1.0\end{array}$ \\
\hline \multicolumn{2}{|c|}{ Effect of $T_{3}$ and $T_{4}$ on synthesis and release of TSH } \\
\hline $\begin{array}{l}g_{53} \\
g_{23} \\
g_{54} \\
g_{24}\end{array}$ & $\begin{array}{l}-0.3 \\
-0.1 \\
-0.3 \\
-0.1\end{array}$ \\
\hline \multicolumn{2}{|c|}{ Effect of iodine on synthesis of $T_{3}$ and $T_{4}$} \\
\hline $\begin{array}{l}g_{36} \\
g_{46}\end{array}$ & $\begin{array}{l}1.0 \\
1.0\end{array}$ \\
\hline \multicolumn{2}{|l|}{ Substrates' kinetic-orders } \\
\hline $\begin{array}{c}h_{11}, h_{22} \\
g_{25} \\
h_{33}, h_{44}\end{array}$ & $\begin{array}{l}1.0 \\
0.02 \\
0.5\end{array}$ \\
\hline \multicolumn{2}{|l|}{ External signals } \\
\hline $\begin{array}{l}g_{57} \\
g_{18}\end{array}$ & $\begin{array}{l}0.1 \\
0.5\end{array}$ \\
\hline
\end{tabular}

release. No changes in $T_{3}$ and $T_{4}$ are detected. The thyroid hormone levels are extremely low in patients showing primary hypothyroidism. In such cases, the TRH test produces TSH levels higher than those observed in normal subjects.

Primary hyperthyroidism is simulated by increasing the initial values of $T_{3}$ and $T_{4}$ (10 and 20 times higher than the normal values). In such a case, the turnover number of thyroid hormones is very low. We have considered a value 0.005 for the relative turnover numbers $F_{3} / F_{2}$ and $F_{4} / F_{2}$ in this kind of patients. The simulation of a typical response to the TRH test is shown in Figure $4 \mathrm{~b}$. 

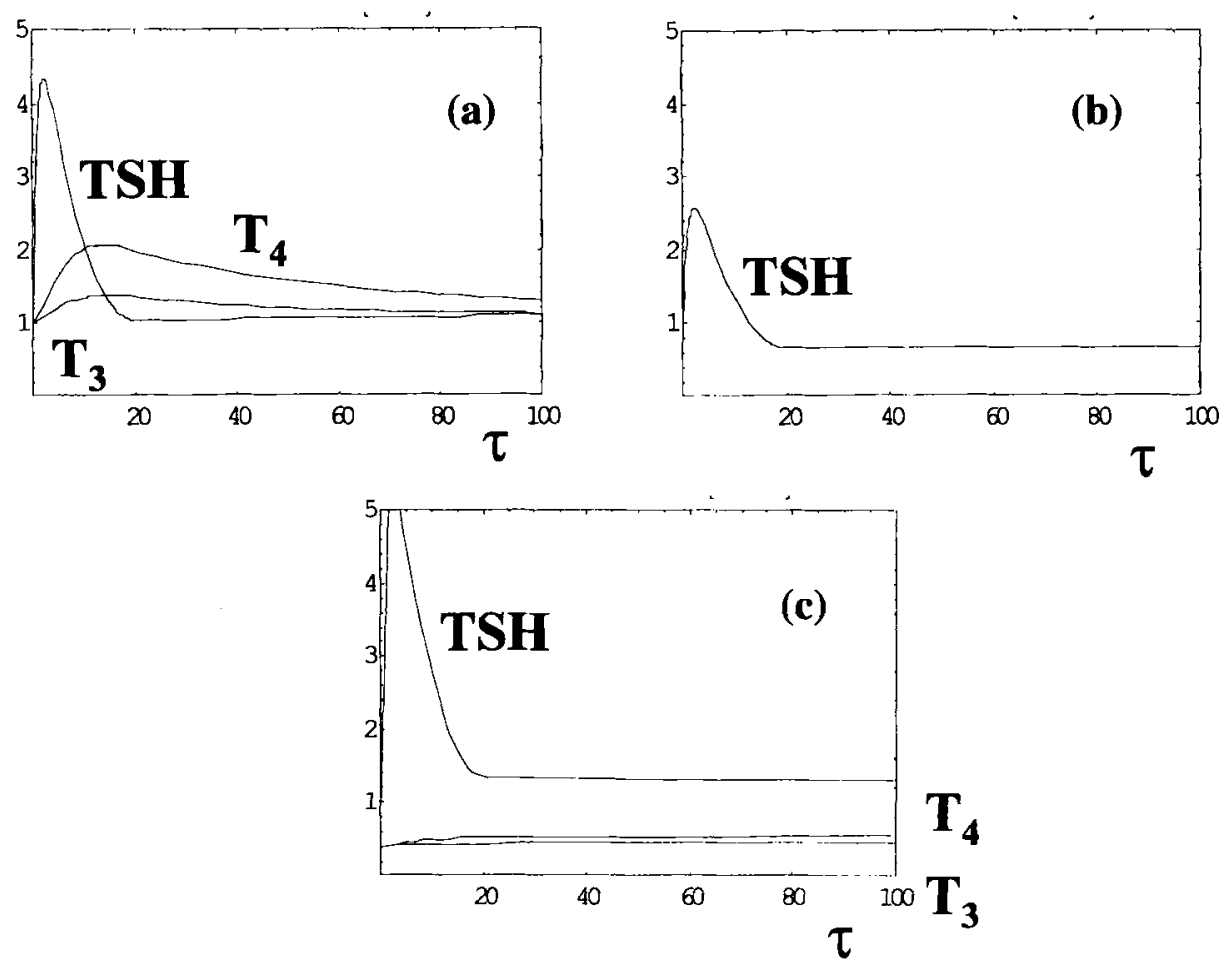

FIGURE 4 Simulated results from applying the TRH test to different patients. Parameter values are indicated in Table I. Except when indicated all the variables have an initial value equal to 1 . The TRH injection is simulated by setting $u_{1}(0)=10^{7}$; (a) Normal subjects; (b) Hyperthyroid patients: $F_{3} / F_{2}=0.005 ; F_{4} / F_{2}=0.005 ; u_{3}(0)=10 ; u_{4}(0)=20 ;$ (c) Hypothyroid patients: $F_{3} / F_{2}=0.005$; $F_{4} / F_{2}=0.005 ; f_{66}=0.1 ; f_{86}=0.1 ; u_{6}(0)=0.01 . T_{3}$ and $T_{4}$ levels are not shown in case (b) to keep the same scale in all cases. Their values after TRH injection are almost unchanged in this condition.

We obtain the expected increase in TSH, with a maximum around two times higher than the basal level. Thyroid hormone levels do not appreciably change in this simulation (not shown in the figure).

Primary hypothyroidism is simulated by lowering the initial value of $T_{3}$ and $T_{4}$ ( $40 \%$ below the normal values). Because primary hypothyroidism can be related to a dysfunction in iodine incorporation into thyroid hormones, we have considered a constitutive low level of iodine for these patients and a weak stimulatory effect on iodine on $T_{3}$ and $T_{4}$ synthesis. Results shown in Figure 4c qualitatively reproduce the clinical observations in this kind of patients.

\subsection{Response of Normal Subjects to Cyclic Patterns of External Signals}

Circadian rhythms are observed as patterns of variation of thyroid hormones (Brabant et al, 1990b,
Custro and Scaglione, 1980; Campos-Barros et al, 1997). Such patterns can be originated by the intrinsic properties of the system, and by the effect of cyclic patterns on the external signals. In Figure 5 we show the model response to a cyclic external signal acting on TRH synthesis as a function of the stimulatory strength measured by the corresponding kinetic-order. Coupling between signal and hormone variation can be observed in this simulation. Similar patterns are obtained by changing the amplitude and frequency of the input signal, which result in concomitant changes in the amplitude and frequency of thyroid hormones (data not shown).

\subsection{Response of Normal Subjects to Chaotic Patterns of External Signals}

Uncontrolled signaling can be the origin of pathological situations. For instance, a continuous stimulation 


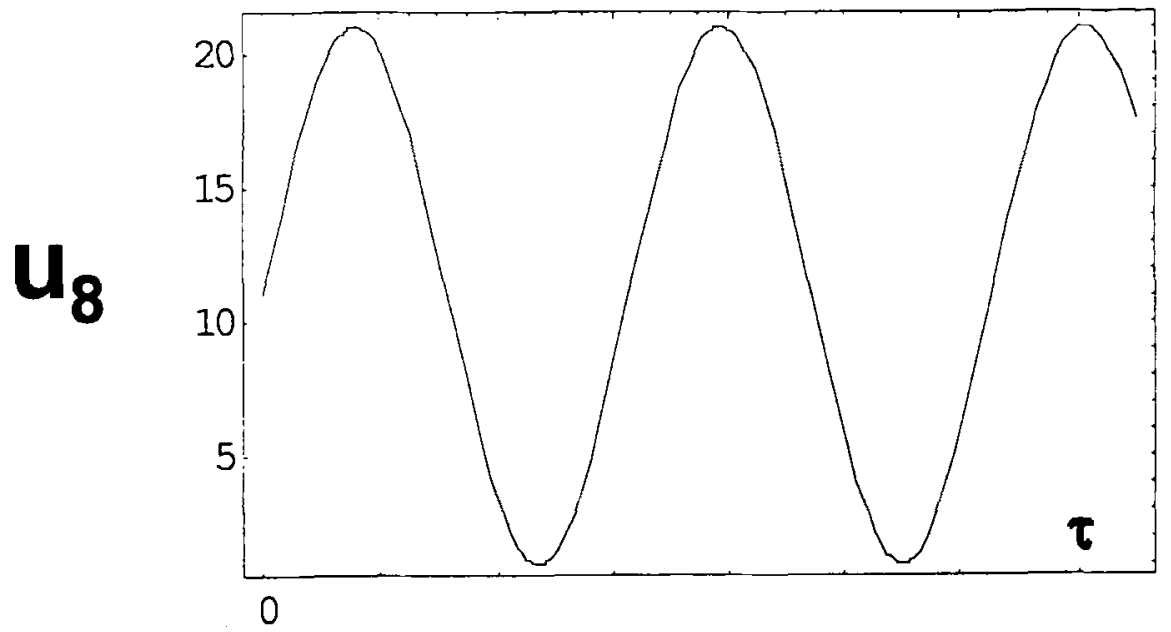

(a)

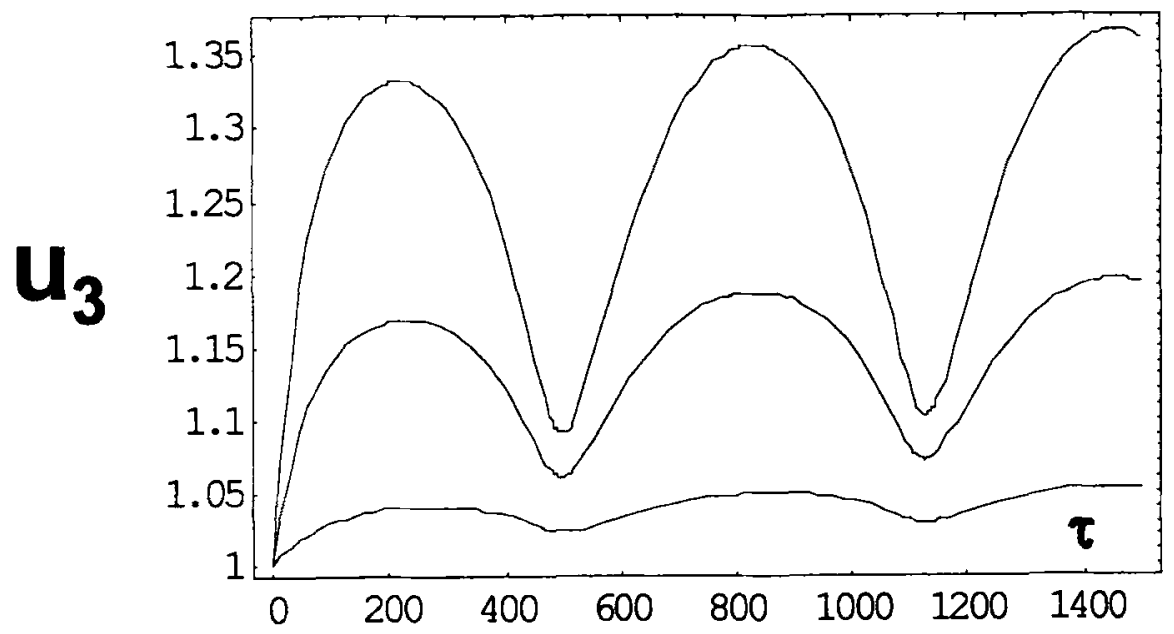

FIGURE 5 Thyroid hormone dynamic behavior simulated by coupling a cyclic stimulus in the synthesis of TRH. The cyclic signal is simulated according to Equation (17-18) using $a=1, b=100, c=0.1$. Model parameters correspond to a normal subject (Table 1).

of TRH by external signals may result in a situation in which TSH cannot return to its normal values, producing an accumulation of thyroid hormones in the long term. We have simulated this situation by using the spike-like dynamic resulting from Equation 14 as a pattern for $u_{8}$ in Equation 8. This leads to a chaotic stimulatory signal for TRH synthesis in our model. The results appear in Figure 6. We observe a chaotic spike-like pattern for TRH resulting from the signal pattern. As expected, this produces a chaotic pattern for TSH that tends to increase its mean value with time, and results in an accumulation of $\mathrm{TSH}_{i}$. As a resulting of the pattern observed for TSH, $T_{4}$ shows a clear tendency to accumulate with time. If this situation is maintained, due to the lack of control in the external signal, a hyperthyroidism may result after some time.

This simulation shows the importance of considering the dynamic behavior as a fundamental part of analyzing a physiological system. In Figure 6, a pathological situation is obtained only by changing the dynamics of the stimulatory signal. No change has been considered in the internal structure of the system, and a normal stimulus would produce a non-pathological pattern. Steady-state analysis of the model would hardly been able of predict this situation. 

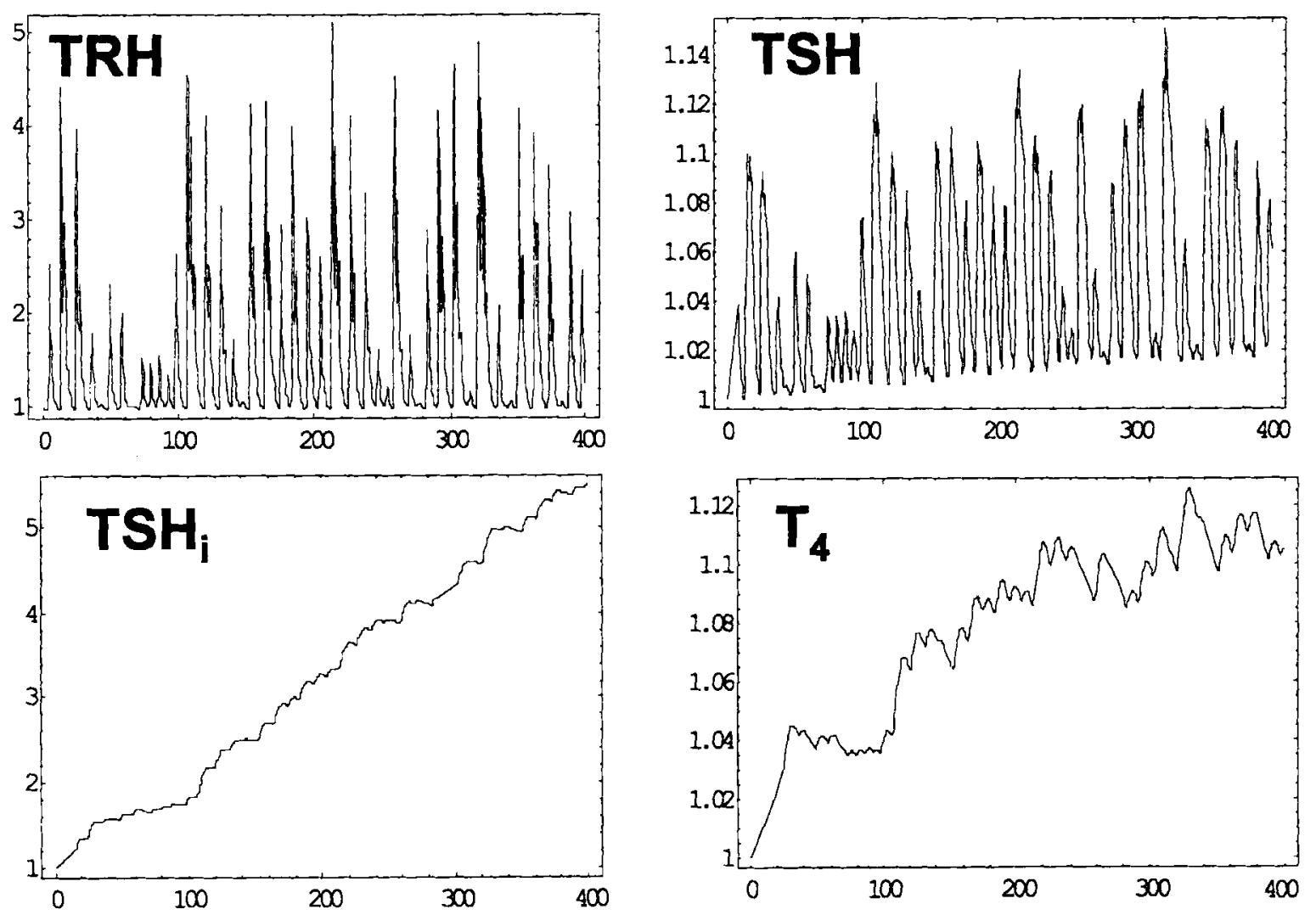

FIGURE 6 Simulation of TRH, TSH and thyroid hormone patterns as a result of a spike-like stimulation of TRH synthesis. As expected, the chaotic signal induces a chaotic pattern in the dependent variables of the model. As a resulting of the perturbation, thyroid hormone accumulates with time.

\section{DISCUSSION}

Clinical observations lead to identify multiple pathological situations in thyroid hormone metabolism. In many instances, these pathological states are related to modified dynamic patterns resulting from changes in the optimal set of interactions within the system. Understanding the origin of such altered patterns is a difficult task that can be oriented by the use of mathematical models consistent with clinical observations. However, the complexity of hormone metabolism makes it difficult to complete such task. A possible strategy consists in developing models that incorporate a complete description of the underlying phenomena. However, besides the difficulty of fully identify such models, the resulting description can be itself too complex for providing an appropriate tool for discussing system's properties.
Mathematical models defined using the powerlaw formalism appear as a compromise between complexity and tractability. Based on a wellestablished approximation of the local processes, power-law models are especially well suited as mathematical descriptions for the whole model. As local approximations, they were not developed as an alternative for in vitro enzyme kinetics. Power-law models focus on the behavior of the whole system. In that sense, since the system constraints the range of variation of the internal variables, the use of an approximated function for the individual processes is not as critical as it may appear. Moreover, it is important to recognize that in many cases no real information exists on the studied processes, making it difficult to select an appropriate function for representing the target process. The power-law modeling allows obtaining a useful representation 
in that case. An example of this situation can be found in aggregated processes that result from the conceptual simplification of the model. Although this is not unique to this approach, the special structure of the power-law equations permits a systematic representation of such processes and the incorporation of different regulatory signals. This strategy provides a final model that can be analyzed both algebraically and by numerical simulations.

An important advantage of such models is that they can be built even on qualitative information, which facilitates the screening of hypothesis on the system structure and regulatory processes. We have illustrated these possibilities in modeling thyroid hormone metabolism. In our example, the suggested model reproduces observations on normal subjects, and hyperthyroid and hypothyroid patients. Furthermore, it can easily incorporate different dynamic patterns for external signals. We have shown how this property can help in investigating the resulting patterns for thyroid hormones. This is an important point, since in many cases pathology is defined as an alteration of the system's dynamic rather than a modification of particular parameters.

The presented model is illustrative and it should be completed in several parts. For instance, iodine metabolism deserves a close look. If we decide to consider different processes at this level, the new model should be modified in an appropriate way. Such modifications can be incorporated in the models without changing the basic mathematical structure. This makes it easier to compare the contribution of the new processes on the whole system behavior. This flexibility is one of the most important advantages of power-law modeling.

\section{APPENDIX 1: A CONCEPTUAL MODEL FOR THE REGULATION OF THYROID HORMONE FORMATION}

The thyroid gland is controlled both by extrinsic and intrinsic mechanisms. The major extrinsic mechanisms are related to the activity of the anterior pituitary, through the action of TSH, and to the dietary iodine. Intrinsic mechanisms are related to self-regulation of iodine intake and hormone release. While the intrinsic mechanisms are difficult to be modeled, TSH variation can be related to the activity of anterior pituitary and its response to extemal factors.

TSH is secreted by thyrotroph cells of the anterior pituitary. This hormone acts upon the thyroid gland stimulating $T_{3}$ and $T_{4}$ secretion. The thyrotroph cells of the anterior pituitary are subjected to controls of opposite sign that modulate its activity. Synthesis and secretion of TSH is inhibited after an increase of $T_{3}$ and $T_{4}$. Alternatively, a deficiency in thyroid hormones produces a simultaneous increase on the synthesis and secretion of TSH. In absence of pathological situations, the principal and most frequent cause of a $T_{3}$ and $T_{4}$ deficit is the insufficient intake of iodine. These observations suggest a negative feedback by the thyroid hormones. The suppressor action of the thyroid hormones is directly performed in the thyrotroph cells of the anterior pituitary.

The principal stimulating control of TSH levels is mediated by the thyrotropin-releasing hormone (TRH). The TRH is released by the hypothalamus, and it reaches the thyrotroph cells through the hypothalamic pituitary carrier vessels. In the surface of thyrotroph cells there are membrane receptors for the TRH. A second messenger mediates the TRH effect, and stimulates the secretion and synthesis of TSH. The levels of thyroid hormones $T_{3}$ and $T_{4}$ have an inhibitory effect on this process regulating the number of TRH receptors (Bauer, 1987). The effects of thyroid hormones on the hypothalamus are unclear, and they are not included in our model.

Other hypothalamic factors (dopamine, somatostatine, etc.) may perform negative effects upon this system, but these effects are weaker comparing to the stimulatory effect of TRH (Scanlon et al., 1980). Hormones other than the thyroid hormones (glycocorticoids, estrogen) may affect the synthesis of the TSH in the thyrotroph cell. Quantitatively, these additional regulators play a minor part when compared with the role of $T_{4}$ and $T_{3}$.

External regulatory stimuli enclose the hypothalamic factors that influence TRH and the signals that 
influence TSH. The number of such external influences and their complex patterns are determinant for the system's dynamics. The great variety of possible patterns for such signals makes it difficult to systematically exploring all the possibilities. As an illustration of the capabilities of the power-law modeling strategy, we have modeled different situations to evaluate their contribution to the dynamic behavior of thyroid hormones.

Iodine is necessary for the synthesis of thyroid hormones. The thyroid gland can regulate its iodine intake and its hormone synthesis even in absence of TSH. This mechanism of thyroid self-modulation allows regulating the secretion of thyroid hormones in front of an abrupt increase of the iodine availability. However, due to the complexity of iodine regulation, iodine will be considered at a constant level in the different simulations.

The conceptual model shown in Figure 1a collects all these observations. It contains two different pools for TSH: one internal to the anterior pituitary gland $\left(\mathrm{TSH}_{i}\right)$, and the other corresponding to the secreted levels of TSH. The model includes negative feedback regulation of thyroid hormone on the synthesis and release of TSH, and the activation of this process by TRH. The effect of TSH on the thyroid gland has been modeled as a stimulation of the $T_{3}$ and $T_{4}$ synthesis. In the final scheme, some of the processes represent an aggregation of several underlying steps. This allows a conceptual simplification that retains the main regulatory influences. For instance, synthesis of TSH comprises several steps, and the influence of TRH and thyroid hormones act at different levels of this process. This is represented as a single process that is affected by these signals. As another example, iodine follows a complex network of reactions before being incorporated into thyroid hormones. This has been reduced to stimulation on thyroid hormones' synthesis.

\section{APPENDIX 2: S-SYSTEM PARAMETER VALUES}

Parameter values on the thyroid hormone system are difficult to estimate, and intersubject variation results in different estimations in similar conditions. Furthermore, different metabolic states correlate with changes in such parameters, making it difficult to define a representative set. Previous models have considered a classical compartmental analysis approach, and linear kinetics is assumed for many of the involved processes (Hatakeyama and Yagi, 1985). This assumption largely reduces the number of parameters to be determined. While this is a legitimate approach, it is interesting to investigate other possibilities related to the relaxation of such hypothesis. The power-law formalism allows for exploring more complex kinetics.

The general meaning of this approach has been discussed in the text. However, before discussing the rationale behind selecting a specific parameter set, it is useful to recall some of the properties of this representation. As a main characteristic, this modeling strategy considers that any of the involved processes can be represented as a function $f\left(X_{1}, \ldots, X_{n}\right)$. Since the functional form is unknown, a Taylorseries approximation in logarithmic space can be used to approximate the actual function. This produces a power-law representation of the form:

$$
V_{i}^{+}=\alpha_{i} \Pi_{j=1}^{n+m} X_{j}^{g_{i j}}
$$

As local sensitivities, kinetic-orders $g_{i j}$ have an intuitive interpretation. If the target process is almost saturated by $X_{j}$ at the selected operating point, the corresponding kinetic-order will be closed to 0 . First-order processes have a kinetic-order equal to 1 , while values greater than 1 are possible only for cooperative kinetics. The term $X_{j}^{g_{i j}}$ can be interpreted as the percent change on $V_{i}^{+}$as a result of a percent change in $X_{j}$. Then, if a $10 \%$ change in $X_{j}$ produces a $3 \%$ increase in $V_{i}^{+}$, an appropriate value for $g_{i j}$ would be 0.3 . If $X_{i}$ is an inhibitor, the corresponding kinetic-order will be negative. For instance, if a $25 \%$ increment in $X_{j}$ results in a $10 \%$ inhibition of the considered process, the corresponding value for the kinetic-order is -0.4 . This intuitive interpretation facilitates to discuss appropriate parameter values for an S-system model (Curto et al., 1997, 1998). 
This interpretation of the kinetic-order parameters allows for tentative estimations that represent a given situation we wish to explore. For demonstrative purposes, we have selected a parameter set (Table II) according to the following rationale:

$$
\text { THR: } u_{1}^{\prime}=F_{1} / F_{2}\left(u_{8}^{g_{18}}-u_{1}^{h_{11}}\right)
$$

Degradation of TRH has been considered a firstorder process (Hatakeyama and Yagi, 1985), which involves $h_{11}=1$. Because $u_{8}$ refer to any of the external signals, the kinetic-order $g_{18}$ can have different values in function of the specific signal. For simulation purposes, we define a kinetic order $g_{18}=0.5$. This corresponds to a stimulatory signal of moderate intensity.

$$
\text { TSH: } u_{2}^{\prime}=u_{1}^{g_{21} 1} u_{3}^{g_{23}} u_{4}^{g_{24}} u_{5}^{g_{25}}-u_{2}^{h_{22}}
$$

Following previous models, we consider a firstorder process for TSH degradation, i.e. $h_{22}=1$ (Hatakeyama and Yagi, 1985). The release of TSH from the anterior pituitary is a complex process. We have considered that this process is regulated by the balance of TRH stimulation and thyroid hormone inhibition, and that it is almost zero-order with respect $\mathrm{TSH}_{i}\left(g_{25}=0.02\right)$. Stimulation of this process by TRH is indicated by $g_{21}$. A value of $g_{21}=0.1$ seemed appropriate if we consider that TRH increases many times over its basal level after a positive stimulus acts on the hypothalamus. A higher value for $g_{21}$ would determine an unrealistic activation of TSH synthesis in this model. Although $T_{3}$ and $T_{4}$ can have asymmetric effects on this process, a tentative value of -0.1 seemed indicated for $g_{23}$ and $g_{24}$. The effect of thyroid hormones and TRH at this level is a variation of previous models. For example, Hatakeyama and Yagi (1985) consider the release of $\mathrm{TSH}$ a first-order process with respect $\mathrm{TSH}_{i}$.

$\mathrm{TSH}_{i}$ :

$$
u_{5}^{\prime}=F_{5} / F_{2}\left(u_{1}^{g_{51}} u_{3}^{g_{53}} u_{4}^{g_{54}} u_{7}^{g_{57}}-u_{1}^{g_{21}} u_{3}^{g_{23}} u_{4}^{g_{24}} u_{5}^{g_{25}}\right)
$$

The stimulatory effect of TRH on the synthesis of $\mathrm{TSH}_{i}$ is related to the value of $g_{51}$. We have found no evidence to sustain the chose of a particular value for this parameter. A tentative value of $g_{51}=0.5$ indicates a $50 \%$ activation after a $100 \%$ increase in TRH. This seems a reasonable choice provided the changes in TRH after stimulation of its synthesis. Activation by external signals is represented by $g_{57}$. Considering that we are building up a parameter set for demonstrative purposes we chose a value of $g_{57}=0.1$, which corresponds to a weak stimulatory signal. Other choices are possible if we want to indicate stronger activation. Finally, the feedback by $T_{3}$ and $T_{4}$ is associated to $g_{53}$ and $g_{54}$. A value of -0.3 for these parameters indicates that the effect of these hormones is more important at this level than at the level of TSH release. These values have no direct relationship with previous models, and represent a hypothesis on the effect of thyroid hormones on $\mathrm{TSH}_{i}$ synthesis.

$$
\begin{aligned}
& T_{3}: u_{3}^{\prime}=F_{3} / F_{2}\left(u_{2}^{g_{32}} u_{6}^{g_{36}}-u_{3}^{h_{33}}\right) \\
& T_{4}: u_{4}^{\prime}=F_{4} / F_{2}\left(u_{2}^{g_{42}} u_{6}^{g_{46}}-u_{4}^{h_{44}}\right)
\end{aligned}
$$

The model of Hatakeyama and Yagi (1985) consider first-order processes for degradation of thyroid hormones. This would correspond to $h_{33}$ and $h_{44}$ equal 1. In our model, we will consider a value of 0.5 indicating an enzymatic process close to half saturation. This implies a slower degradation of thyroid hormones, which result in a slightly longer effect of these hormones. The stimulatory effect of TSH on the synthesis of $T_{3}$ and $T_{4}$ correspond to $g_{32}$ and $g_{42}$. As a hypothesis, we have considered a slightly higher effect on the synthesis of $T_{4}\left(g_{42}=1\right.$ versus $g_{32}=0.5$ ). Since iodine is considered at a constant level, the parameter values given to $g_{36}$ and $g_{46}$ are irrelevant.

Relative turnover numbers:

Relative turnover numbers have been adjusted after several runs to qualitatively reproduce the observed clinical results for the TRH test in normal subjects. Their values are shown in Table II.

\section{APPENDIX 3: STEADY-STATE HORMONAL RESPONSE TO EXTERNAL SIGNALS}

The steady-state response to an external perturbation can be characterized by logarithmic-gains. A 
logarithmic-gain is a global sensitivity that quantifies the effect of a change in an independent variable on a dependent variable. They can be interpreted as logarithmic derivatives and are easily computed in a power-law model, either in S-system or GMA form. If the model is an S-system, logarithmic-gains can be computed algebraically from the steady-state solution. (Savageau, 1972; Savageau and Sorribas, 1989; see Cascante et al. (1995) for an explanation on how to compute these gains). For instance, the response to a sustained increase in $X_{8}$ on the levels of TSH can be quantified by the corresponding logarithmic-gain. This corresponds to the derivative of $\operatorname{Ln}\left(X_{2}\right)$ with respect $\operatorname{Ln}\left(X_{8}\right)$ on the steady-state solution of Equation 6:

$$
\begin{aligned}
& \frac{\mathrm{d} \operatorname{Ln}\left(X_{2}\right)}{\mathrm{d} \operatorname{Ln}\left(X_{8}\right)}=L_{28} \\
& \quad=\frac{g_{51} g_{18} h_{33} h_{44}}{\left[\left(h_{22} h_{33}-g_{53} g_{32}\right) h_{44}-g_{54} h_{33} g_{42}\right] h_{11}}(15)
\end{aligned}
$$

This gain is expected to be positive if $X_{8}$ is a positive stimulus ( $g_{18}$ positive), and negative if it is inhibitory ( $g_{18}$ negative). According to the signs of the involved kinetic-orders, the sign of $L_{28}$ in Equation 7 has always the desired value, independently of the particular parameter values. Of course, the value of $L_{28}$ will depend on the specific parameter values.

Following an increment in TSH, we expect a release in both $T_{3}$ and $T_{4}$. An intuitive analysis makes it difficult to check if this will be the outcome in the model. An increase in TSH will activate the release of $T_{3}$ and $T_{4}$. Then, an increase in $T_{3}$ and $T_{4}$ will inhibit both the synthesis and the release of TSH, which will result in a decrease both in $T_{3}$ and in $T_{4}$. Following this reasoning, it is difficult to say if $T_{3}$ and $T_{4}$ will increase or not after an increase in TRH due to a response to external activation. The expected behavior can be discussed after obtaining the corresponding logarithmic-gains. In this case, the response of $T_{3}$ and $T_{4}$ to a positive signal on TRH can be expressed by the logarithmic-gains $L_{37}$ and $L_{47}$ :

$$
\begin{aligned}
& L_{37}=\frac{g_{32} h_{44} g_{57}}{\left(h_{22} h_{33}-g_{53} g_{32}\right) h_{44}-g_{54} h_{33} g_{42}} \\
& L_{47}=\frac{h_{33} g_{42} g_{57}}{\left(h_{22} h_{33}-g_{53} g_{32}\right) h_{44}-g_{54} h_{33} g_{42}}
\end{aligned}
$$

These gains are always positive if the external signal is positive, and negative if the external signals are inhibitory. This agrees with our knowledge on thyroid hormone metabolism. Again, the magnitude of the change will depend on the values of the kinetic-orders, and the difference on the increment in $T_{3}$ and $T_{4}$ will be determined by the values of $g_{32} h_{44}$ and $h_{33} g_{42}$. We expect a large logarithmic-gain, i.e. a several fold increases in $T_{3}$ and $T_{4}$, for positive signals. According to Equation 16, a constraint for a high logarithmic-gain in the case of $L_{37}$ and $L_{47}$ is:

$$
\begin{aligned}
& g_{32} h_{44} g_{57} \gg \\
& \quad\left(h_{22} h_{33}-g_{53} g_{32}\right) h_{44}-g_{54} h_{33} g_{42} \\
& h_{33} g_{42} g_{57} \gg \\
& \quad\left(h_{22} h_{33}-g_{53} g_{32}\right) h_{44}-g_{54} h_{33} g_{42}
\end{aligned}
$$

Thus, a low inhibitory effect of $T_{3}$ and $T_{4}$ on the synthesis of TSH $\left(g_{53}\right.$ and $\left.g_{54}\right)$, and a low kinetic order of TRH on its own degradation $\left(h_{22}\right)$ are critical for obtaining a high logarithmic-gain. Alternatively, a high effect of the stimulatory signals $\left(X_{7}\right)$ on the production of $\mathrm{TSH}_{\mathrm{i}}\left(V_{5}^{+}\right)$will result in a favorable condition for a high logarithmic-gain.

In the case of a perturbation in the signals that affect the synthesis of TRH, the conditions are:

$$
\begin{aligned}
& g_{51} g_{32} h_{44} g_{18} \gg \\
& \quad\left[\left(h_{22} h_{33}-g_{53} g_{32}\right) h_{44}-g_{54} h_{33} g_{42}\right] h_{11} \\
& g_{51} h_{33} g_{42} g_{18} \gg \\
& \quad\left[\left(h_{22} h_{33}-g_{53} g_{32}\right) h_{44}-g_{54} h_{33} g_{42}\right] h_{11}
\end{aligned}
$$

From these equations, additional conditions for high logarithmic-gains are a high kinetic-order of TRH on the synthesis of TSH, and a strong effect of $X_{8}$ on the synthesis of TRH. 


\section{References}

Bauer, K. (1987) Adenohypophyseal degradation of thyrotropin releasing hormone regulated by thyroid hormones. Nature, 330, 375-377.

Brabant, G., Ocran, K., Ranft, U., von zur Muhlen, A. and Hesh, R. D. (1989) Physiological regulation of thyrotropin. Biochemie, 71, 293-301.

Brabant, G., Frank, K., Ranft, U., et al. (1990a) Physiological regulation of circadian and pulsatile thyrotropin secretion in normal man and women. Journal of Clinical Endocrinology and Metabolism, 70, 403-409.

Brabant, G., Prank, K., Ranft, U. et al. (1990b) Circadian and pulsatile TSH secretion under physiological and pathophysiological conditions. Hormone and Metabolic Research Supplement Series, 23, 12-17.

Cascante, M., Curto, R. and Sorribas, A. (1995) Comparative characterization of the fermentation pathway of Saccharomyces cerevisiae using Biochemical Systems Theory and Metabolic Control Analysis: Steady-state analysis. Mathematical Biosciences, 130, 51-69.

Campos-Barros, A., Musa, A., Flecher, A., Hessenius, C., Gaio, U., Meinhold, H. and Baumgartner, A. (1997) Evidence for circadian variations of thyroid hormone concentrations and type UU 5'-iodothyronine deiodinase activity in the rate central nervous system. Journal of Neurochemistry, 68, 795-803.

Curto, R., Sorribas, A. and Cascante, M. (1995) Comparative characterization of the fermentation pathway of Saccharomyces cerevisiae using Biochemical systems Theory and Metabolic Control Analysis: Model definition and nomenclature. Mathematical Biosciences, 130, 25-50.

Curto, R., Voit, E. O., Sorribas, A. and Cascante, M. (1997) Validation and steady-state analysis of a power-law model of purine metabolism in man. Biochemical Joumal, 324, 761 - 775 .

Curto, R., Voit, E. O., Sorribas, A. and Cascante, M. (1998) Mathematical models of purine metabolism in man. Mathematical Biosciences, 151, 1-49.

Custro, N. and Scaglione, R. (1980) Circadian rhythm of TSH in adult men and women. Acta Endocrinologica Copenhagen, 95, 465-471.

Hlavacek, W. S. and Savageau, M. A. (1996) Rules for coupled expression of regulator and effector genes in inducible circuits. Journal of Molecular Biology, 255, 115-125.

Hatakeyama, T. and Yagi, H. (1985) Computer simulation for hormones related to primary thyropathy. Biological Cybernetics, 52, 259-266.

Hays, M. T., Broome, M. R. and Turrel, J. M. (1988) A multicompartmental model for iodine, Thyroxine and Triiodothyronine metabolism in normal and spontaneously hyperthyroid cats. Endrocrinology, 122, 2444-2461.

Jackson, I. M. (1982) Thyrotropin releasing hormone. New England Journal of Medicine, 306, 145-155.

Jacobs, G., Whittem, T., Sams, R., Calvert, C. and Ferguson, D. (1997) Pharmacokinetics of propanolol in healthy cats during euthyroid and hyperthyroid states. American Journal of Veterinarian Research, 58, 398-403.

Lin, C. and Segel, L. (1974) Mathematics applied to deterministic problems in the natural sciences. MacMillan, New York

Leproult, R., Van Reeth, O., Byrne, M. M., Sturis, J. and Van Cauter, E. (1997) Sleepiness, performance, and neuroendocrine function during sleep deprivation: effects of exposure to bright light or exercise. Journal of Biological Rhythms, 12, 245-258.
Magner, J. A. (1990) Thyroid-stimulating hormone: biosynthesis, cell biology and bioactivity. Endocrine Reviews, 11, 354-385.

Mendel, C. M. (1989) The free hormone hypothesis: A physiologically based mathematical model. Endocrine Reviews, 10, $232-274$.

Morley, J. E. (1981) Neuroendocrine control of thyrotropin secretion. Endocrine Reviews, 2, 396-436.

Ni, T.-C. and Savageau, M.A. (1996a) Model assessment and refinement using strategies from biochemical systems theory: application to metabolism in red blood cells Journal of Theoretical Biology, 179, 329-368.

Ni, T.-C. and Savageau, M. A. (1996b) Application of biochemical system theory to metabolism in human red blood cells. Signal propagation and accuracy of representation Journal of Biological Chemistry, 271, 7927-7941.

Oppenheimer, J. H., Schwartz, H. L., Mariash, C. N., Kinlaw, W. B., Wong, N. C. W. and Freake, H. C. (1987) Advances in our understanding of thyroid hormone action at the cellular level. Endocrine Reviews, 8, 288-308.

Rondanelli, M., Solerte, S. B., Fioravanti, M., Scevola, D., Locatelli, M., Nimoli, L. and Ferrari, E. (1997) Circadian secretory pattern of growth hormone, insulin-like growth factor type I, cortisol, adrenocorticotropic hormone, thyroid-stimulating hormone, and prolactin during HIV infection. AIDS Research and Human Retroviruses, 13, $1243-1249$.

Savageau, M. A. and Sorribas, A. (1989) Constraints Among Molecular and Systemic Properties: Implications for Physiological Genetics. Journal of Theoretical Biology, 141, 93-115.

Savageau, M. A. (1995a) Michaelis-Menten mechanism reconsidered: Implications of fractal kinetics. Joumal of Theoretical Biology, 176, 115-124.

Savageau, M. A. (1972) The behavior of intact biochemical control systems. Current Topics in Cellular Regulation, 6, $63-130$.

Savageau, M. A. (1992) Critique of the enzymologists test tube in Foundations of Medical Cell Biology. Bittar, E. E. (ed) Jai Press Inc, Greenwich, Connecticut, 45-108

Savageau, M. A. (1995b) Enzyme kinetics in vitro and in vivo: Michaelis-Menten revisited in Principles of Medical Biology. Bittar, E. E. (ed) JAI Press Inc., Greenwich. Connecticut, 93-146.

Scanlon, M. F, Lewis, M., Weightman, D. R., Chan, V and Hall, R. (1980) The neuroregulation of human thyrotropin secretion in Frontiers in neuroendocrinology. Martini, L, Ganong, W. F. (eds). Raven Press, New York.

Shiraishi, F. and Savageau, M. A. (1992a) The Tricarboxilic Acid Cycle in Dictiostelium discoideum.I Formulation of the alternative kinetic representations. Journal of Biological Chemistry, 267, 22912-22918.

Shiraishi, F. and Savageau, M. A. (1992b) The Tricarboxilic Acid Cycle in Dictiostelium discoideum.II Evaluation of model consistency and robustness. Journal of Biological Chemistry, 267, 22919-22925.

Shiraishi, F. and Savageau, M. A. (1992c) The Tricarboxilic Acid Cycle in Dictiostelium discoideum.III Analysis of steadystate and dynamic behavior. Journal of Biological Chemistry, 267, 22926-22933.

Shiraishi, F. and Savageau, M. A. (1992d) The Tricarboxilic Acid Cycle in Dictiostelium discoideum.IV Resolution of discrepancies between alternative methods of analysis. Journal of Biological Chemistry, 267, 22934-22943. 
Sorribas, A. and Savageau, M. A. (1989) A comparison of variant theories of intact biochemical systems: 1. Enzymeenzyme interactions and Biochemical Systems Theory. Mathematical Biosciences, 94, 161-193.

Sorribas, A., Curto, R. and Cascante, M. (1995) Comparative characterization of the fermentation pathway of Saccharomyces cerevisiae using Biochemical Systems Theory and Metabolic Control Analysis: Model validation and dynamic behavior. Mathematical Biosciences, 130, 71-84.

Sullivan, P. F., Wilson, D. A., Mulder, R. T. and Joyce, P. R. (1997) The hypothalamic-pituitary-thyroid axis in, major depression. Acta Psychiatrica Scandinavica, 95, 370-378.
Voit, E. O. (Ed.) (1991) Canonical Nonlinear Modeling: Ssystem Approach to Understanding Complexity. Van Nostrand Reinhold, New York.

Voit, E. O. and Savageau, M. A. (1987) Accuracy of alternative representations for Integrated Biochemical Systems. Biochemistry, 26, 6869-6880.

Voit, E. O. (1993) S-system modeling of complex systems with chaotic input. Environmetrics, 4, 153-186.

Yamada, K., Distefano, J. J. $3^{\text {rd }}$, Yen, Y. M. and Nguyen, T. T. (1996) Steady-state regulation of whole-body thyroid hormone pool sizes and interconversion rates in hypothyroid and moderately T3-stimulated rats. Endocrinology, 137, 5624-5633. 


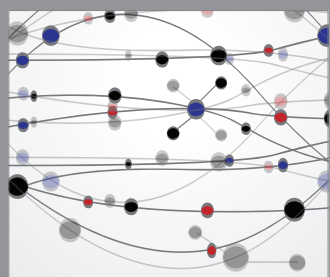

The Scientific World Journal
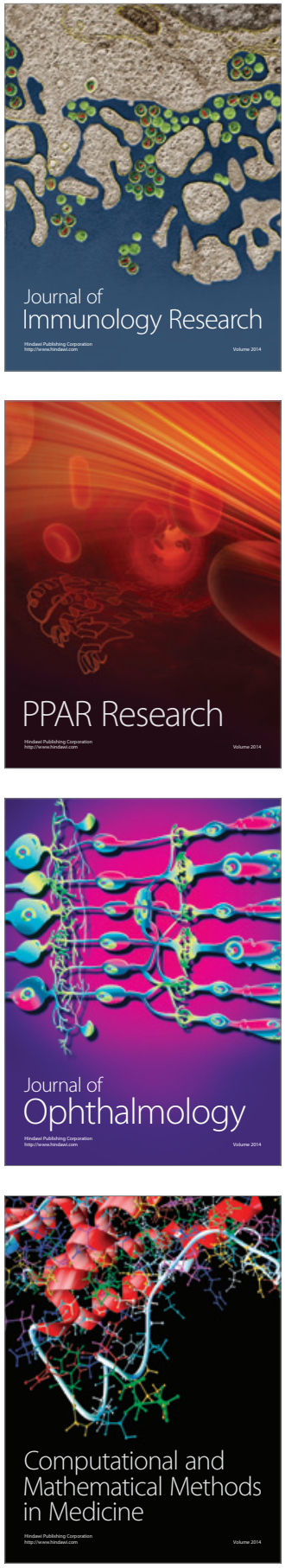

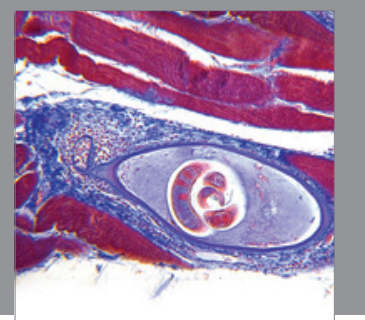

Gastroenterology

Research and Practice
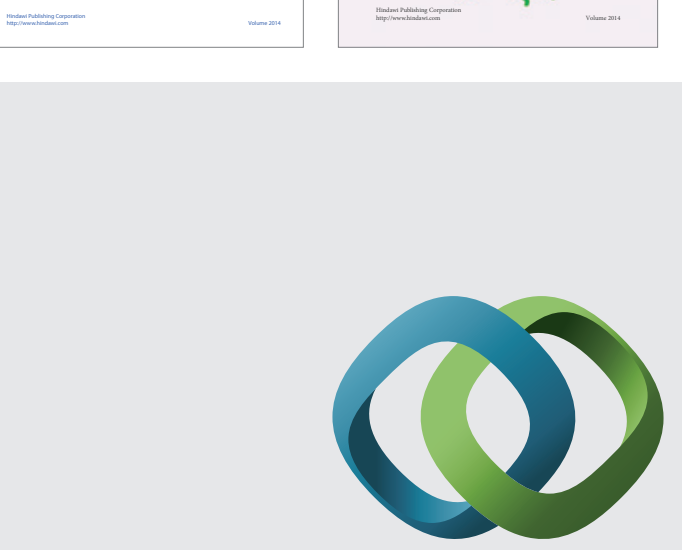

\section{Hindawi}

Submit your manuscripts at

http://www.hindawi.com
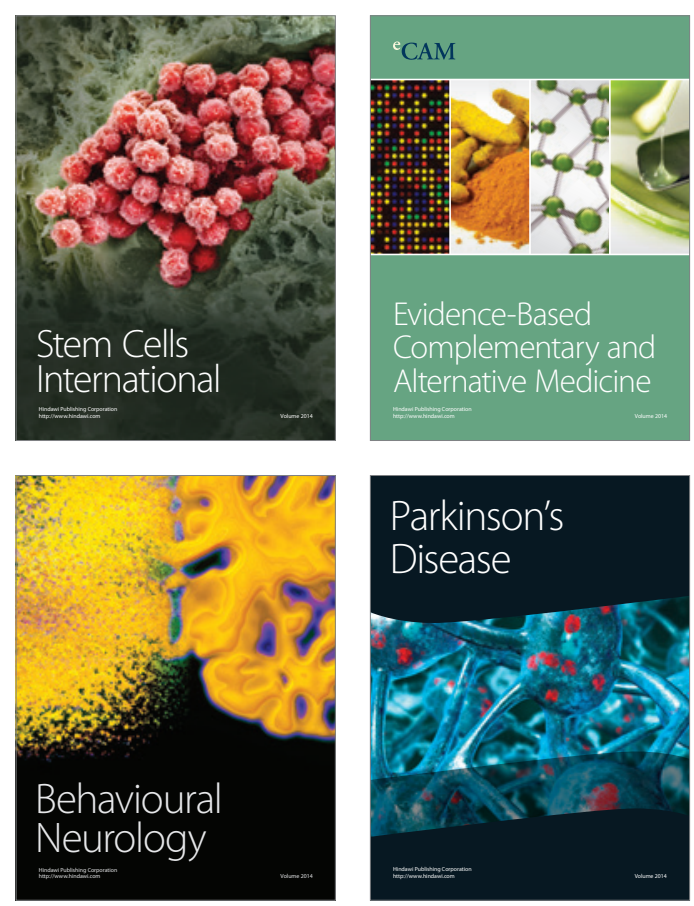

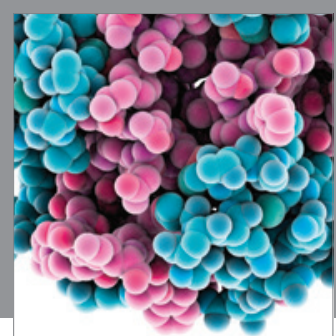

Journal of
Diabetes Research

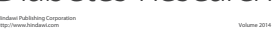

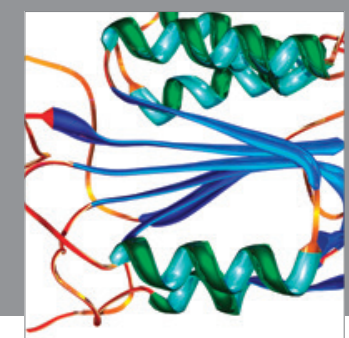

Disease Markers
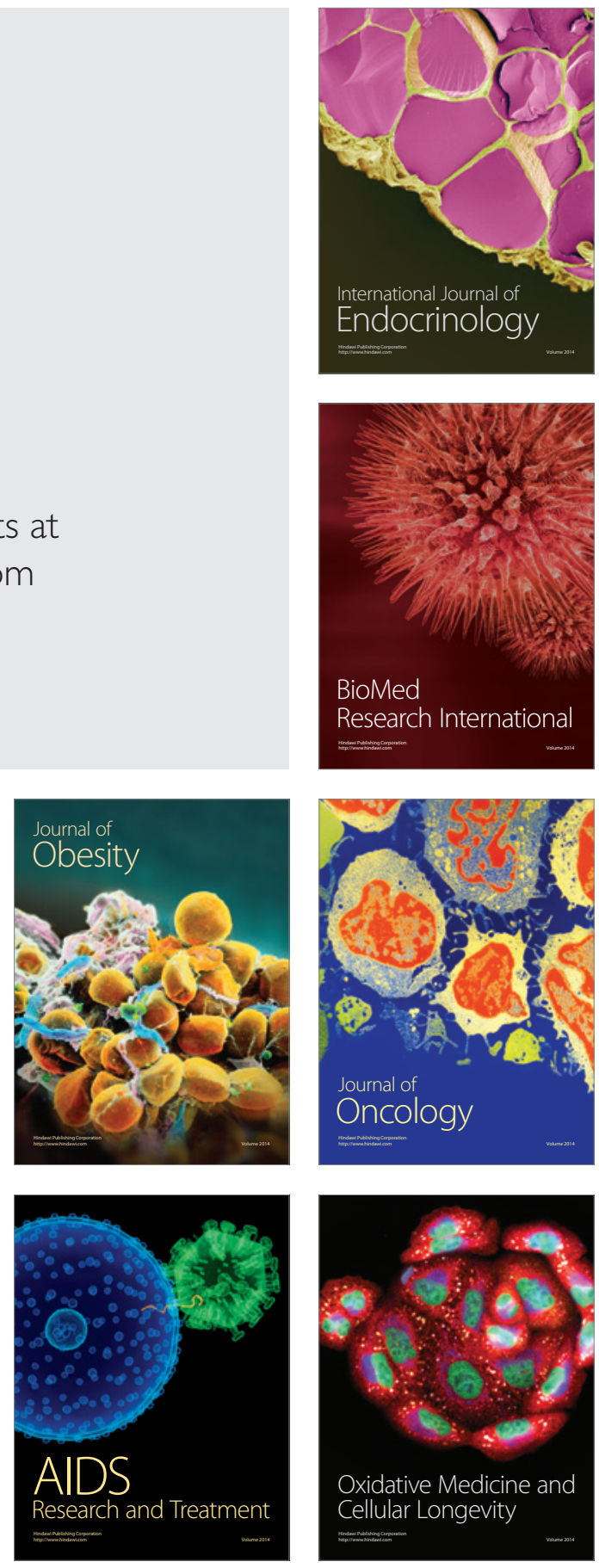